Compact Superconducting Final Focus Magnet Options for the ILC

\author{
B. Parker, M. Anerella, J. Escallier, M. Harrison, P. He, A. Jain, \\ A. Marone, K.C. Wu (BNL) \\ T. Markiewicz, T. Maruyama, Y. Nosochkov, and A. Seryi (SLAC)
}

May 16, 2005

Superconducting Magnet Division

\author{
Brookhaven National Laboratory \\ Operated by \\ Brookhaven Science Associates \\ Upton, NY 11973 \\ Under Contract with the United States Department of Energy \\ Contract Number DE-AC02-98CH10886
}




\section{DISCLAIMER}

This report was prepared as an account of work sponsored by an agency of the United States Government. Neither the United States Government nor any agency thereof, nor any of their employees, nor any of their contractors, subcontractors or their employees, makes any warranty, express or implied, or assumes any legal liability or responsibility for the accuracy, completeness, or any third party's use or the results of such use of any information, apparatus, product, or process disclosed, or represents that its use would not infringe privately owned rights. Reference herein to any specific commercial product, process, or service by trade name, trademark, manufacturer, or otherwise, does not necessary constitute or imply its endorsement, recommendation, or favoring by the United States Government or any agency thereof or its contractors or subcontractors. The views and opinions of authors expresses herein do not necessarily state to reflect those of the United States Government or any agency thereof. 


\title{
COMPACT SUPERCONDUCTING FINAL FOCUS MAGNET OPTIONS FOR THE ILC*
}

\author{
B. Parker", M. Anerella, J. Escallier, M. Harrison, P. He, A. Jain, A. Marone and K.C. Wu \\ BNL, Upton, NY 11973, U.S.A. \\ T. Markiewicz, T. Maruyama, Y. Nosochkov and A. Seryi, SLAC, Menlo Park, CA 94025, U.S.A.
}

Abstract

The QD0 quadrupole, the final focus magnet closest to the Interaction Point (IP) for the ILC $20 \mathrm{mr}$ crossing angle layout, must provide strong focusing yet be adjustable to accommodate collision energy changes for energy scans and low energy calibration running. It must also be compact to allow disrupted beam and Beamstrahlung coming from the IP to pass outside into an independent instrumented beam line that leads to a high-power beam absorber. In designing QD0 we take advantage of recent $\mathrm{BNL}$ experience making direct wind superconducting magnets. Here we review important considerations for the present design, report on progress producing a prototype, describe our compact quadrupole concept in greater detail, and relate this work to other $\mathrm{LC}$ design challenges.

\section{EVOLUTION OF VERY COMPACT COILS}

The compact QD0 superconducting magnet design builds upon previous BNL work making direct wind superconducting magnets for the HERA-II and BEPC-II upgrades[1]; however, QD0 for the ILC $20 \mathrm{mr}$ crossing angle layout requires a focusing gradient, $144 \mathrm{~T} / \mathrm{m}$, that is about an order of magnitude greater than those for HERA$\mathrm{II}$ and BEPC-II. Fortunately the $10 \mathrm{~mm}$ radius QD0 aperture is correspondingly smaller so its peak field is of similar magnitude. Our first challenge was then to show that producing such small coils was feasible.

To this end we made test windings using single-strand, $0.33 \mathrm{~mm}$ diameter, $\mathrm{NbTi}$ wire ending with a $1.6 \mathrm{~m}$ long three-layer prototype being wound. This coil was intended to be used as an insertion inside a second larger diameter quadrupole planned to be made using seven-strand round cable (six $0.33 \mathrm{~mm}$ strands twisted around one central strand) at the previously demonstrated cable winding bend radius. The outer quadrupole coil was specified to provide $\approx 60 \%$ of the QD0 operating gradient.

In order to develop magnets allowing upgrade of $a \approx 25$ $\mathrm{mr}$ crossing angle $\mathrm{e}^{+} \mathrm{e}^{-} \mathrm{IP}$ to do $\gamma$ collisions, we looked to make QD0 even more compact. Compactness is critical due to two features of Compton backscattering (used to make the $\gamma \mathrm{s}$ ), the spent electron spectrum fills $a \approx 10 \mathrm{mr}$ cone and has a low-energy tail extending down to $8 \mathrm{GeV}$ (compared to $250 \mathrm{GeV}$ primary). The large aperture

\footnotetext{
"This manuscript has been authored by Brookhaven Science Associates, LLC under Contract No. DE-AC02-98CH1-886 with U.S. Department of Energy. The United States Government retains, and the publisher, by accepting article for publication, acknowledges, a world-wide license to publish or reproduce published form of this manuscript, or allow others to do so, for the United states Government purposes.

"parker@bnl.gov
}

magnet used to compensate QD0's external fringe field overlapped QD0 in our original coil design.

We were pleased to demonstrate automatic winding with $\approx 1 \mathrm{~mm}$ seven-strand cable at the same tight bend radius that we earlier used for winding the $1.6 \mathrm{~m}$ singlestrand prototype. Using cable for the innermost coil windings, in place of single-strand wire, improves the packing factor sufficiently to dispense with the inner/outer nested two-coil structure. We can have a single very compact multi-layer coil made just with cable.

The design has evolved in another way. For the original NLC beam parameters, QD0 vibration stability was a concern. After adoption of cold linac beam parameters by the ILC parameters subgroup, we find that QD0 vibration limits are relaxed somewhat by using beam deflection feedback techniques over the course of the long ILC bunch train. Nevertheless because we want to set aside uncertainties whether normal liquid helium, in either two phase or single phase flow, might provide a source term for internal vibration, we have decided to pursue using pressurized He-II at $1.9^{\circ} \mathrm{K}$ to cool QD0. Another advantage with He-II cooling is increased thermal reserve (for stability against energy deposition transients).

Based upon $1.9^{\circ} \mathrm{K}$ quench measurements of wire used for the seven-strand cable, our final design is compact enough to place a second independent (separate cryostat) magnet, QEX, along side QD0 on the outgoing beam as in Fig.1. In addition to quadrupole coils, QEX and QD0 have dipole, skew-dipole and skew-quadrupole correction coils.

For the extraction line this configuration allows:

- local compensation of QD0's external field,

- earlier focusing to reduce extraction line apertures,

- better capture of low energy secondaries (deflected crossing solenoid field lines) via a D-focusing QEX,

- and orbit steering to keep beam hitting the same spot on absorber after changing the primary beam energy.

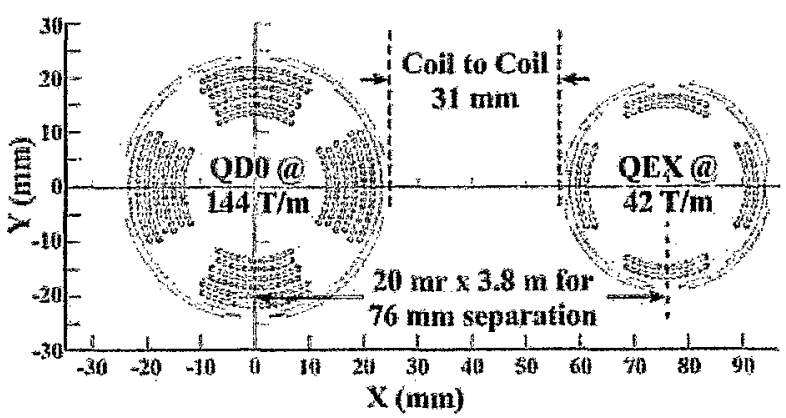

Figure 1. Cross section for side-by-side QD0 and QEX magnet coils at a distance of $3.8 \mathrm{~m}$ from the IP. 


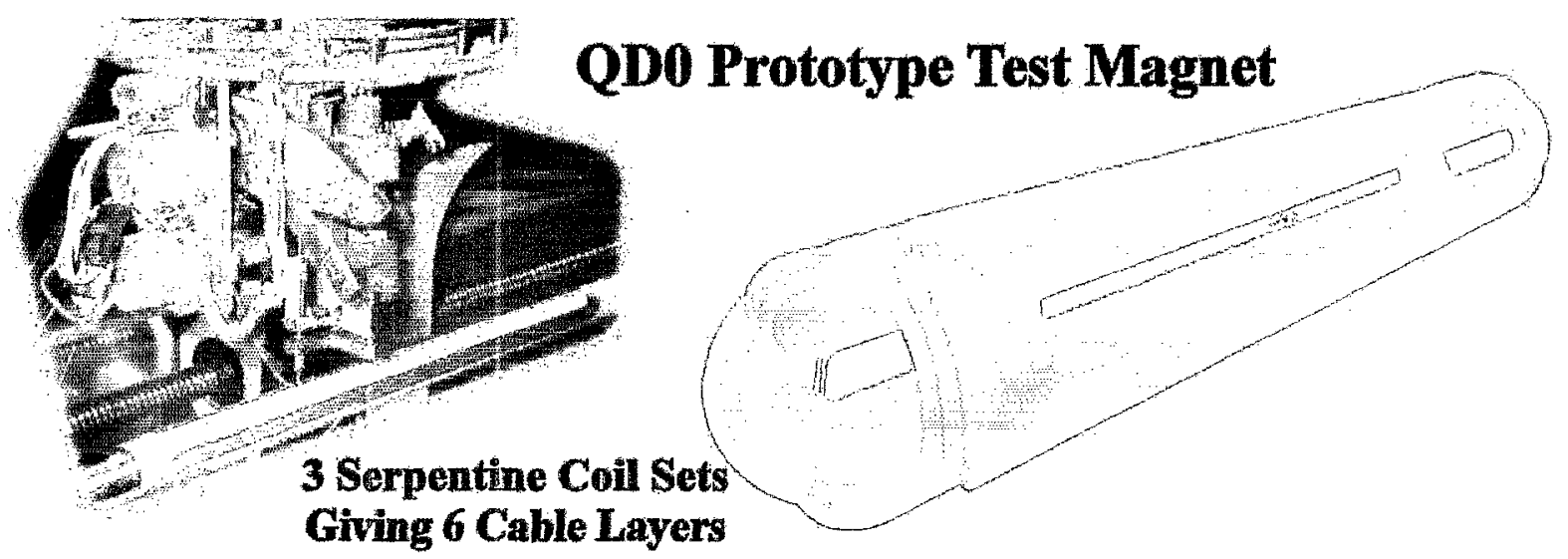

Figure 2. Start of winding for QD0 Prototype Test Magnet, QT, along with 3D view of the final coil configuration.

\section{PRODUCING A QD0 TEST PROTOTYPE}

At this time we are midway through production of the QD0 test magnet prototype, QT, shown in Fig.2 using BNL Direct Wind coil construction technology. When complete, this magnet will have three two-layer Serpentine style[2] coil sets for a total of six cable winding layers. While conductor layout in both the QT body and ends is identical to what would be used for a full length, $\approx 2 \mathrm{~m}$ QDO, we have carefully chosen its $380 \mathrm{~mm}$ (15 inch) pattern length to maximize the information we can gain testing it using existing equipment. The $1.6 \mathrm{~m}$ single-strand coil gave valuable feedback on our winding process but it is too long to fit the small laboratory dewar, with special test capabilities, that we now prefer to use.

QT is long enough for us to measure body harmonics in the center of the magnet (conductor placement accuracy indicator) independent of its integral field and end field harmonics; yet it is compact enough to fit inside an existing small test dewar that is outfitted with an $8 \mathrm{~T}$

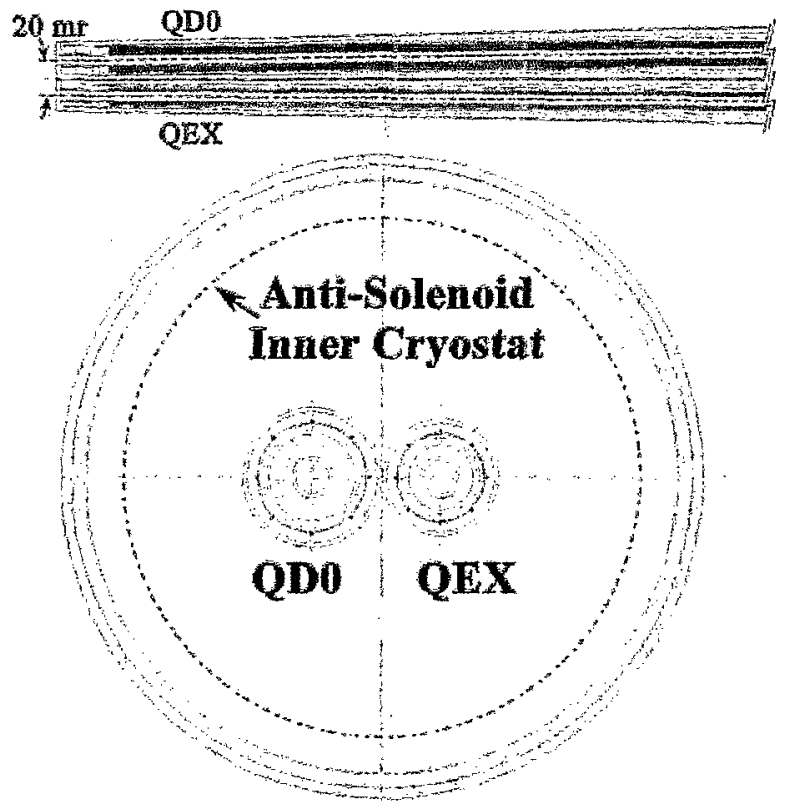

Figure 3. Side-By-Side QD0/QEX tapered cryostats, plan view (above) and section view in Anti-Solenoid (below). solenoid. Using 1.8:1 Cu:SC seven-strand round cable the present $\mathrm{QD} 0 / \mathrm{QT}$ design at $1.9^{\circ} \mathrm{K}$ in a $3 \mathrm{~T}$ solenoidal (detector fringe) field for $144 \mathrm{~T} / \mathrm{m}$ has the operating current at $\approx 65 \%$ of the short sample limit predicted from wire measurements. At $4.2^{\circ} \mathrm{K}$, but no background field, the same current is $\cong 100 \%$ short sample. Finally at $1.9^{\circ} \mathrm{K}$, in order to reach interesting coil Lorentz force levels without having to run at excessively high currents, we plan to raise the background field to about $7 \mathrm{~T}$ to force a quench at operating current. QT will also have a full complement of spot heaters and voltage taps for our quench propagation studies at lower currents and fields.

\section{ILC 20 MR CROSSING ANGLE DESIGN}

The dual side-by-side cryostat configuration is shown in Fig.3. The round cryostat outer envelope maintains a taper along its length in order to fit the tight IP end spacing. Consistent with this taper the QD0/QEX heat shields and cold masses step up while going away from the $\mathrm{IP}$. Heat exchange is done between pressurized $\mathrm{He}-\mathrm{II}$ in contact with the coils and a low pressure He-II bath.

A QD0 mid cryostat cross section at an internal cold mass support location is shown in Fig.4. Budgeted space for He-II is sufficient to transport $15 \mathrm{~W}$ to the heat exchanger at negligible temperature difference; however, sections closer to the IP have less space for HE-II and only pass $4 \mathrm{~W}$ under these conditions. We shall consider using a more complicated elliptical cold mass cross section at the IP end to provide additional space for $\mathrm{He}$-II.

We place anti-solenoid coils, used for detector solenoid

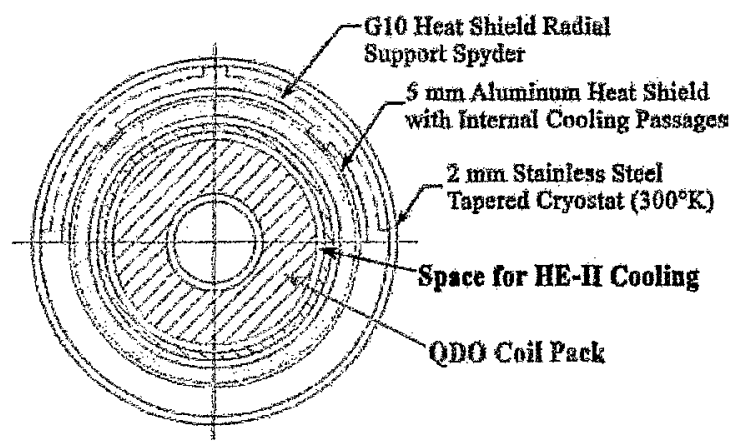

Figure 4. QD0 Cryostat Detail at Support Location. 


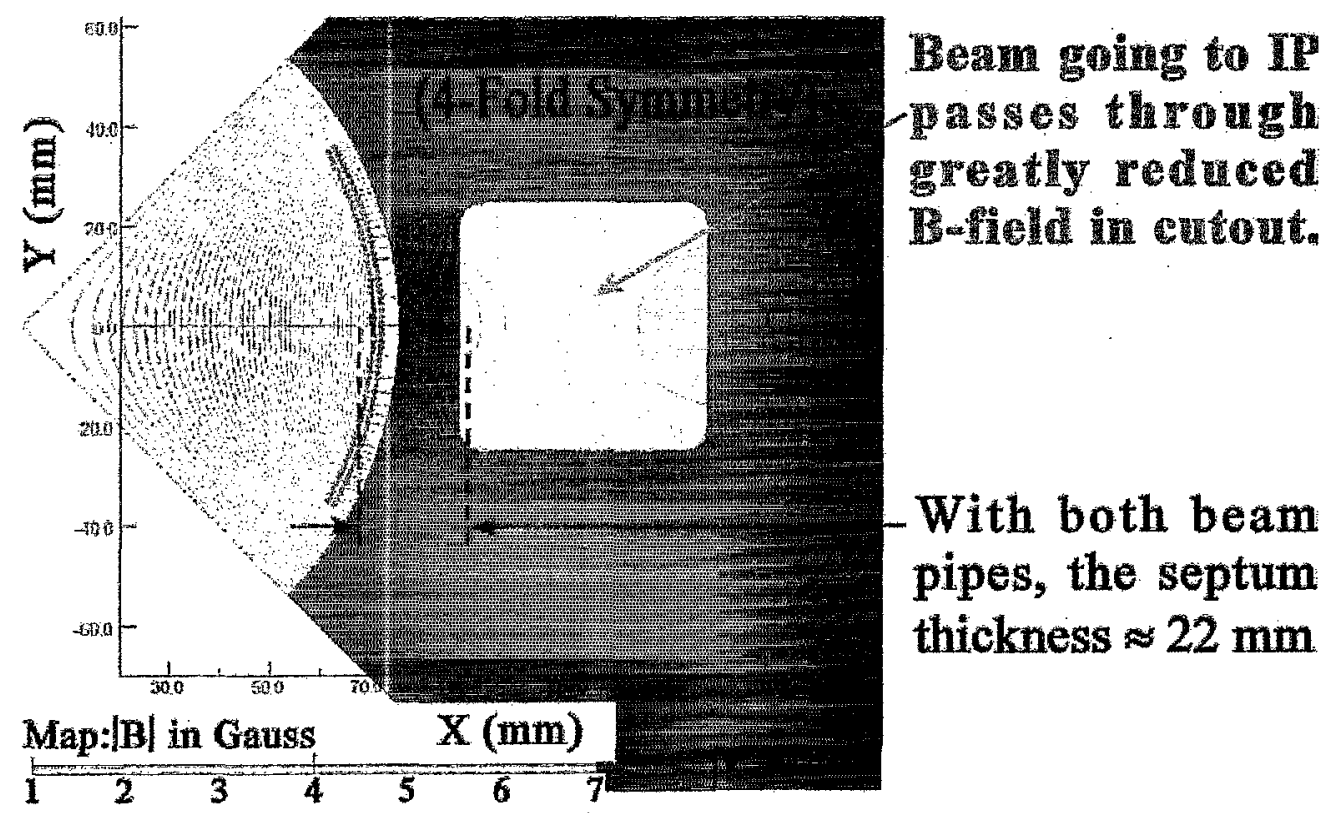

Figure 6. Super Septum Quadrupole with $|\mathrm{B}|$ map in cutout region. Model assumes 4-fold symmetry. compensation[3], in an independent cryostat because we do not want to take the strong longitudinal repulsion the anti-solenoid experiences via the QD0 cold mass but would rather pass it directly to the detector yoke as indicated in Fig.5. Having to pass large forces in the final focus magnets would complicate dramatically the design of their support structure and was judged to make feedback based cryostat stabilization more difficult.

The present $20 \mathrm{mr}$ final focus optics has $\mathrm{L}^{*}, \mathrm{QD} 0$ to IP distance, of $3.51 \mathrm{~m}$. The tentative budget for the cryostat must accommodate a warm-to-cold transition, BPMs, collimators and backscatter masks and we expect to do fine tuning of this design at a future date. With $\mathrm{LC}$ beam power $\approx 10 \mathrm{MW}$, the beam delivery system must be designed so that beam loss in magnets is tolerable and the extraction line must transport highly disrupted beam cleanly to the absorber. Energy deposition is important to the design and operation of superconducting magnets and preliminary tracking estimations of this are encouraging, but significant beam loss is found from radiative Bhabha scattering. Detailed calculations are now in progress.

Like QD0, the other final focus doublet magnet, QF1, the associated sextupoles, SD0 and SF1 and the first few extraction line quadrupoles are direct wind construction. But outside the detector solenoid field these coils are surrounded by magnetic yokes to gain transfer function and eliminate beam line cross talk.

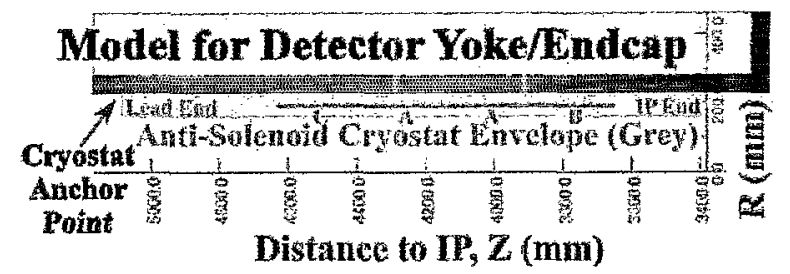

Figure 5. Anti-Solenoid, installed inside the detector yoke, feels $\approx 15$ Ton longitudinal force directed away from the IP and therefore needs to be well anchored.

Similar magnets may prove useful for the $2 \mathrm{mr}$ crossing angle layout (with tight beam separation extending much further from the IP). For small separation we have the super septum quadrupole, shown in Fig.6, with a cutout yoke. The $2 \mathrm{mr}$ layout design studied so far has low gradient extraction quadrupoles but with large apertures to accept Beamstrahlung and a wide energy spread. Low field in the cutout at the incoming beam location limits synchrotron radiation background and emmitance dilution effects. Minimal cold magnetic yoke material is sufficient to have the cutout region field about a few Gauss in magnitude. With a two-layer direct wind coil a narrow septum is realized. But kilowatt level energy deposition is possible in upstream and downstream magnets and right now it is unclear if practical collimators and septum masks exist to ensure tolerable super septum quadrupole energy deposition if it were to be used in the $2 \mathrm{mr}$ layout.

\section{SUMMARY}

Compact magnets are needed for the ILC beam delivery and extraction systems; direct wind production matches their requirements quite well. A QD0 test prototype is being made that soon will be tested in background field to simulate influence of a detector solenoid. Feedback from these tests will provide input to the very active ongoing optimization of the $20 \mathrm{mr}$ crossing angle design.

\section{REFERENCES}

[1] P. Wanderer, et. al., "Completion of Superconducting Magnet Production by BNL for HERA Luminosity Upgrade," MT-17, Geneva, Switzerland, Sept., 2001.

[2] B. Parker, TOAA010, these proceedings.

[3] Y. Nosochkov and A. Seryi, "Compensation of detector solenoid effects on the beam size in a linear collider," Phys. Rev. ST Accel. Beams, 8, 021001, 2005. 